\title{
PD-L1 Expression in Patients with Non-small Cell Lung Cancer
}

\author{
KATSUHIRO MASAGO, SHIRO FUJITA, AKITO HATA, CHIYUKI OKUDA, \\ REIKO KAJI, NOBUYUKI KATAKAMI and YUKIO HIRATA \\ Division of Integrated Oncology, Institute of Biomedical Research and Innovation, Kobe, Japan
}

\begin{abstract}
Aim: The aim of this study was to evaluate whether irradiation induces the expression of tumor programed cell death ligand $1(P D-L 1)$ in patients with non-small cell lung cancer (NSCLC). Patients and Methods: Seventeen patients with NSCLC who received chemoradiotherapy and underwent tumor resection and six patients whose pre-treatment biopsy specimens were available, were analyzed by immunohistochemistry for PD-L1 expression between September 2011 and June 2016 at the Institute of Biomedical Research and Innovation Hospital. Results: Among six patients for which pre-irradiation biopsy samples were available, the H-score for $P D-L 1$ was reduced after irradiation following staining with two different antibody clones (SP28-8 and SP142). A PD-L1 $H$-score $>5$ with SP28-8 antibody (hazard ratio $=6.46 ; 95 \%$ confidence interval $=1.209-34.53 ; p=0.029)$ was a significant negative factor for duration of progression-free survival after curative operation or chemoradiation. Conclusion: We showed that tumor PD-L1 expression decreased in patients with NSCLC who received chemoradiotherapy and radiation resistance might be due to pre-treatment PD-L1 expression.
\end{abstract}

Non-small cell lung cancer (NSCLC) is a leading cause of cancer-related death worldwide. Recent discoveries in biomedical research have provided a greater understanding of the druggable molecular basis of this disease (1). However, beyond targeting of these driver mutations, platinum-based, doublet chemotherapy remains the standard therapy.

In recent years, immune checkpoint inhibitors, such as those targeting programmed cell death protein 1 (PD1) or PD-ligand 1 (PD-L1), were introduced as another therapeutic option in this field with promising results (2). A monoclonal antibody against PD1, nivolumab, is now in clinical use and several PD-L1 antibodies are being evaluated in clinical

Correspondence to: Katsuhiro Masago, MD, Ph.D., 2-2 Minatojimaminamimachi, Cyuo-ku, Kobe City, Hyogo 650-0047 Japan. Tel: +81 783065200, Fax: +81 783060729, e-mail: masago@fbri.org

Key Words: Immunohistochemistry, irradiation, non-small cell lung cancer, programmed cell death protein 1 . trials (3). Furthermore, companion biomarkers that can predict the efficacy of these drugs are under exploration, such as immunohistochemistry (IHC) for intra-tumoral/ microenviromental PD1 or PD-L1, infiltrating T-lymphocytes and somatic mutation burden.

Deng $\mathrm{L}$ et al. reported that PD-L1 expression increased on tumor cells $\left(\mathrm{CD} 45^{-}\right)$, dendritic cells $\left(\mathrm{CD} 11 \mathrm{c}^{+}\right)$and macrophages $\left(\mathrm{CD} 11 \mathrm{~b}^{+} \mathrm{F} 4 / 80^{+}\right)$after irradiation with $12 \mathrm{~Gy}$ in a TUBO tumor model, which may weaken irradiationinduced antitumor immunity (4). If this phenomenon is confirmed in the clinical setting, it would provide a rationale for including anti-PD-L1 therapy in the concurrent or adjuvant setting in combination with irradiation for the treatment of patients with NSCLC.

Here, we evaluated PD-L1 status of resected tumor tissue after induction chemoradiotherapy and also compared the PD-L1 status of pre- and post-treatment tumor tissues when specimens were available.

\section{Materials and Methods}

Patients (Cohort 1). Seventeen patients with NSCLC who received chemoradiotherapy and underwent tumor resection (one patient for brain metastasis) and six patients whose pre-treatment biopsy specimens were available were analyzed for PD-L1 IHC between September 2011 and June 2016 at the Institute of Biomedical Research and Innovation Hospital. This study was approved by the Institutional Review Board of our institution (Number 15-32). This study was conducted in accordance with the Declaration of Helsinki.

Cohort 2: Validation cohort. We retrospectively screened electronic medical records of 83 patients with advanced NSCLC who had undergone histological re-biopsies between January 2010 and October 2015 at in our Institute or Kobe City General Hospital.

$P D-L 1 I H C$. Paraffin-embedded tumor tissues were sectioned at a thickness of $4 \mu \mathrm{m}$ and the sections were then mounted on glass slides for PD-L1 immunohistochemistry (IHC). PD-L1 IHC was performed using SP142 and SP28-8 antibodies (Spring Biosciences, Pleasanton, CA, USA) for membranous staining of tumor cells. The H-score method was adopted to evaluate both the percentage and intensity of staining. The semi-quantitative $\mathrm{H}$-score was determined by multiplying the percentage of stained cells by the intensity score ( 0 , absent; 1 , weak; 2 , moderate; and 3 , strong) 


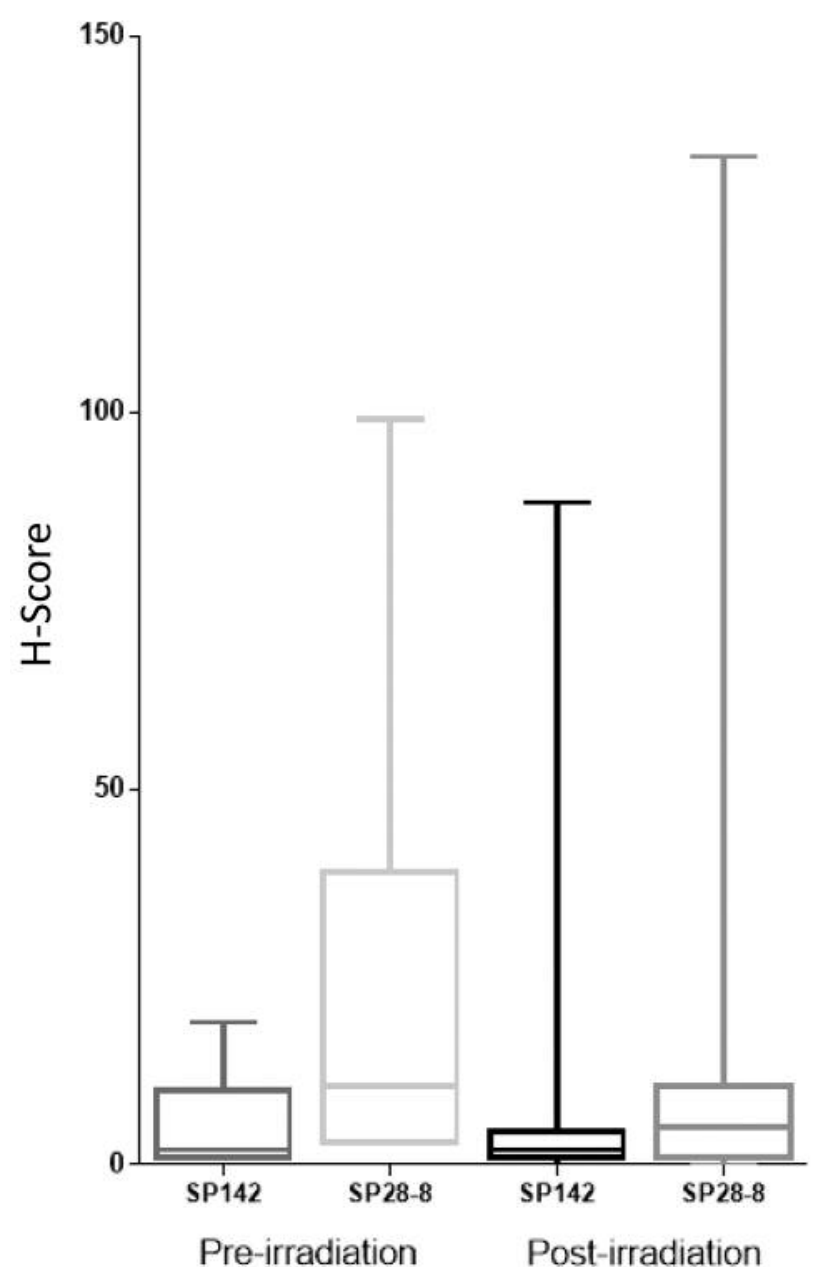

Figure 1. H-Score for programmed cell death ligand 1 (PD-L1) of preirradiation biopsy and post-irradiation resected tumor specimens using two different antibody clones, SP142 and SP28-8. Boxes represent $75 \%$ quartile, median and $25 \%$ quartile. Lines represent upper and lower whiskers.

leading to a maximum value of 300 corresponding to $100 \%$ of tumor cells positive for PD-L1 with an overall staining intensity score of 3 . We did not definePD-L1 expression as positive or negative in this study.

Mutational analysis of the epidermal growth factor receptor $(E G F R)$ and anaplastic lymphoma kinase (ALK) genes. A sufficient number of cancer cells for a pathological diagnosis (i.e. several hundred cells) were obtained from paraffin-embedded biopsy specimens by manual microdissection. Biopsy or surgical specimens were used for analysis of somatic EGFR mutations in exons 18-21, as previously described (5). IHC analysis of $A L K$ expression was performed by fluorescent in situ hybridization with a mouse monoclonal antibody to ALK (ALK1; Dako, Japan), as previously described $(6,7)$. Seventeen patients were analyzed for ALK status, among which two had $A L K$ rearrangements and 15 were wild-type for $A L K$.
Table I. Characteristics of patients of cohort $1(n=17)$.

\begin{tabular}{|c|c|c|}
\hline Characteristic & & Value \\
\hline \multirow[t]{2}{*}{ Age, years } & Range & $44-80$ \\
\hline & Median & 66 \\
\hline \multirow[t]{2}{*}{ Gender, n (\%) } & Male & $11(64.7)$ \\
\hline & Female & $6(35.3)$ \\
\hline \multirow[t]{4}{*}{ Smoking status, n (\%) } & Non-smoker & $3(17.7)$ \\
\hline & Smoker & $14(82.3)$ \\
\hline & Former & $9(52.9)$ \\
\hline & Current & $5(29.4)$ \\
\hline Performance status, n (\%) & $0-1$ & $17(100)$ \\
\hline \multirow[t]{3}{*}{ Tumor histology, n (\%) } & Adenocarcinoma & $11(54.7)$ \\
\hline & Squamous cell carcinoma & $5(29.4)$ \\
\hline & LCNEC & $1(15.9)$ \\
\hline \multirow[t]{4}{*}{ Stage, n (\%) } & IIA & $3(17.7)$ \\
\hline & IIB & $3(17.7)$ \\
\hline & IIIA & $6(35.3)$ \\
\hline & IIIB & $5(29.3)$ \\
\hline \multirow[t]{2}{*}{$E G F R, \mathrm{n}(\%)$} & Exon19 deletion & $2(11.8)$ \\
\hline & Wild-type & $12(70.5)$ \\
\hline \multirow[t]{2}{*}{$A L K$ rearrangement, $\mathrm{n}(\%)$} & Positive & $1(6.0)$ \\
\hline & Negative & $11(64.7)$ \\
\hline \multirow[t]{8}{*}{ PD-L1 H-score } & SP142 & \\
\hline & Range & $0-88$ \\
\hline & Mean & 7.6 \\
\hline & Median & 2 \\
\hline & SP28-8 & \\
\hline & Range & $1-134$ \\
\hline & Mean & 13.0 \\
\hline & Median & 5 \\
\hline
\end{tabular}

LCNEC, Large-cell neuroendocrine carcinoma; EGFR, epidermal growth factor receptor; $A L K$, anaplastic lymphoma kinase; PD-L1, programmed cell death ligand 1; SP142/SP28-8, antibody clone used.

Statistical analysis. Progression-free survival (PFS) was calculated from the date of diagnosis until the date of disease recurrence or death for all enrolled patients. To evaluate risk factors associated with PFS, a Cox proportional hazards regression model was used. PFS curves were generated using the Kaplan-Meier method. The log-rank test was used to evaluate differences between PFS curves. Statistical analyses were performed using GraphPad Prism (ver. 6; GraphPad Software, Inc., San Diego, CA, USA) and 'R' (R Foundation for Statistical Computing, Vienna, Austria) software programs.

\section{Results}

The characteristics of the 17 enrolled patients are shown in Table I. All the patients were Japanese and underwent tumor resection after induction chemoradiotherapy. The patients had a median age of 66 (range $=44-80)$ years and comprised of $11(64.7 \%)$ men and six $(35.3 \%)$ women. Patient characteristics with tumor stage and somatic mutation status are shown in Table I. 

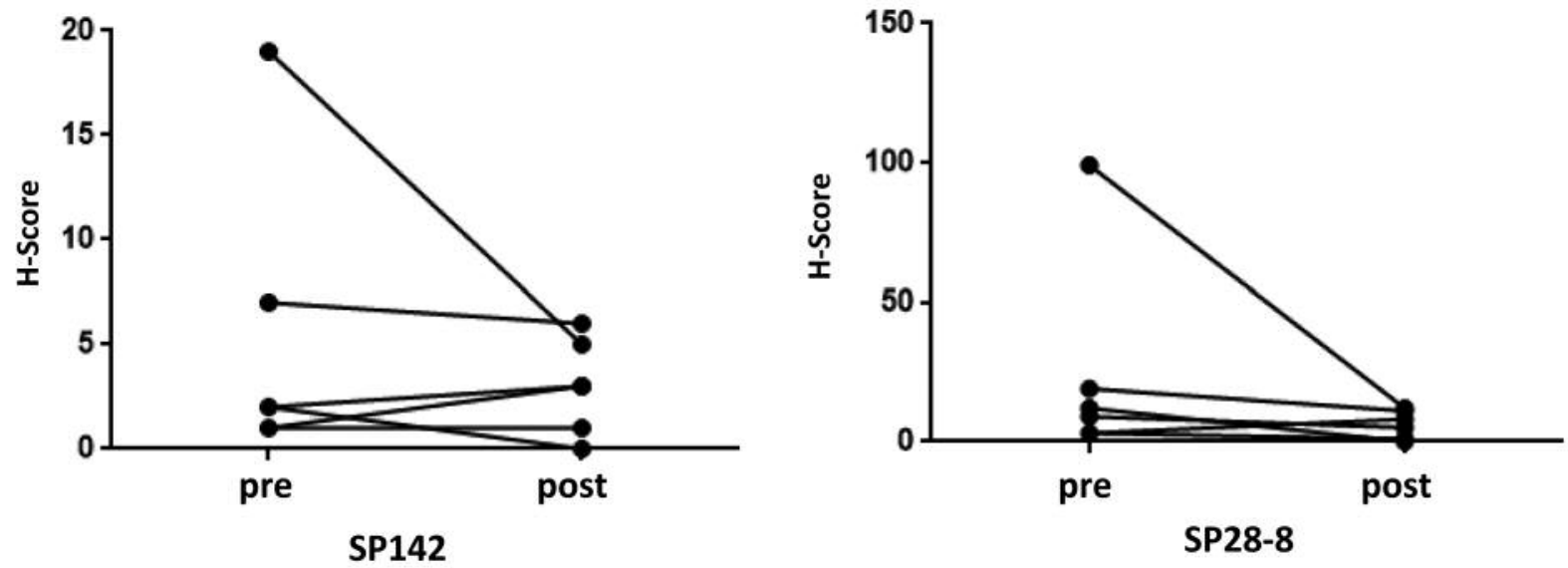

Figure 2. Changes in H-score for programmed cell death ligand 1 (PD-L1) between pre-and post-irradiation samples among six patients using two different antibody clones SP142 (A) and SP28-8 (B).

Table II. Characteristics of patients of Cohort 2 (validation) ( $n=83)$.

\begin{tabular}{llc}
\hline Characteristic & & Value \\
\hline Age, years & Range & $26-84$ \\
& Median & 74 \\
Gender, n (\%) & Male & $40(48.2 \%)$ \\
& Female & $43(51.8 \%)$ \\
Smoking status, n (\%) & Non-smoker & $27(67.5 \%)$ \\
& Smoker & $56(22.5 \%)$ \\
Tumor histology, n (\%) & Adenocarcinoma & $73(89.0 \%)$ \\
& Squamous cell carcinoma & $8(9.6 \%)$ \\
& LCNEC & $2(1.4 \%)$ \\
Genotype, n (\%) & EGFR-mutated & $63(75.9 \%)$ \\
& ALK rearrangement & $3(3.6 \%)$ \\
& Wild-type & $17(20.5 \%)$ \\
PD-L1 H-score & SP142 & \\
& Range & $0-91$ \\
& Mean & 3 \\
& Median & 3 \\
& SP28-8 & $1-150$ \\
& Range & 9 \\
& Mean & 9 \\
& Median & \\
& &
\end{tabular}

LCNEC, Large-cell neuroendocrine carcinoma; EGFR, epidermal growth factor receptor; $A L K$, anaplastic lymphoma kinase; PD-L1, programmed cell death ligand 1; SP142/SP28-8, antibody clone used.

The H-scores for PD-L1 are listed in Table I and Figure 1. The H-score tended to be higher following staining with the SP28-8 clone antibody than with the SP142 clone antibody. The median score (range) of PD-L1 expression was $2(0-88)$ and 5 (1-134) for the SP142 and SP28-8 clones, respectively. Among the six patients for which a preirradiation biopsy sample was available, the H-score for PD-

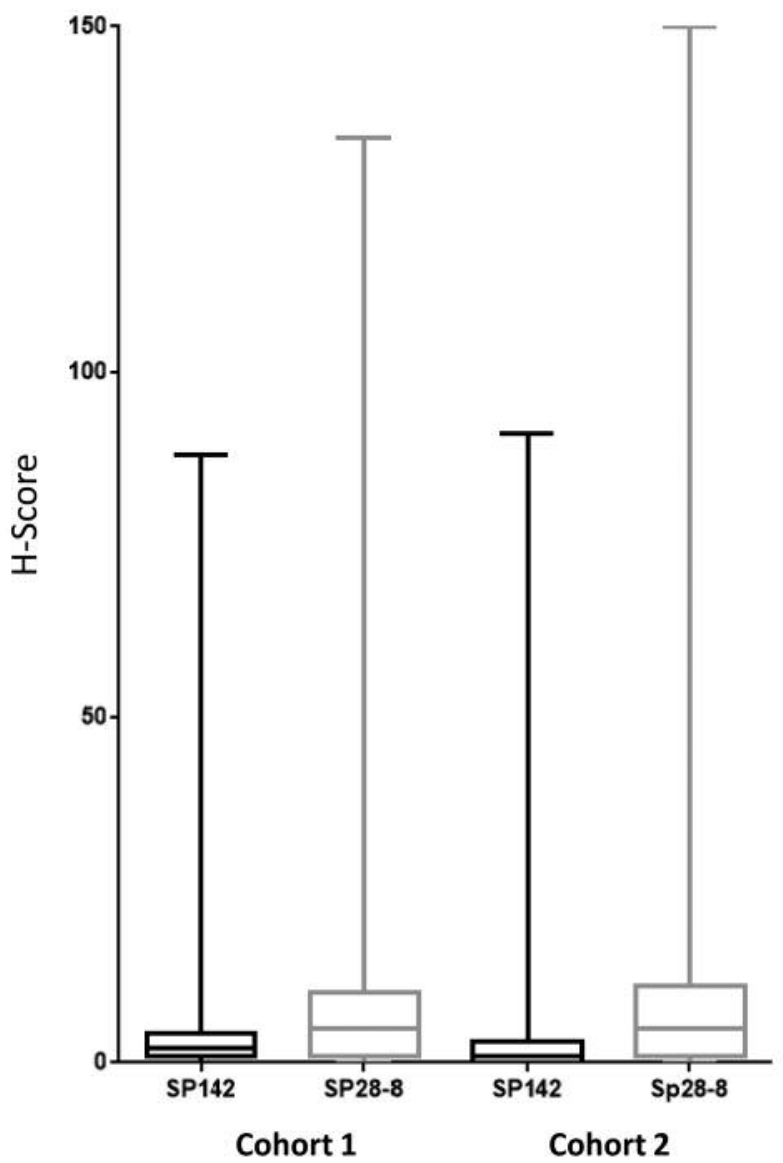

Figure 3. H-Score for programmed cell death ligand 1 (PD-L1) of cohort 1 (post-irradiation biopsy) and cohort 2 using two different antibody clones, SP142 and SP28-8.

L1 was reduced after irradiation, regardless of the antibody clone used (Figure 2). 

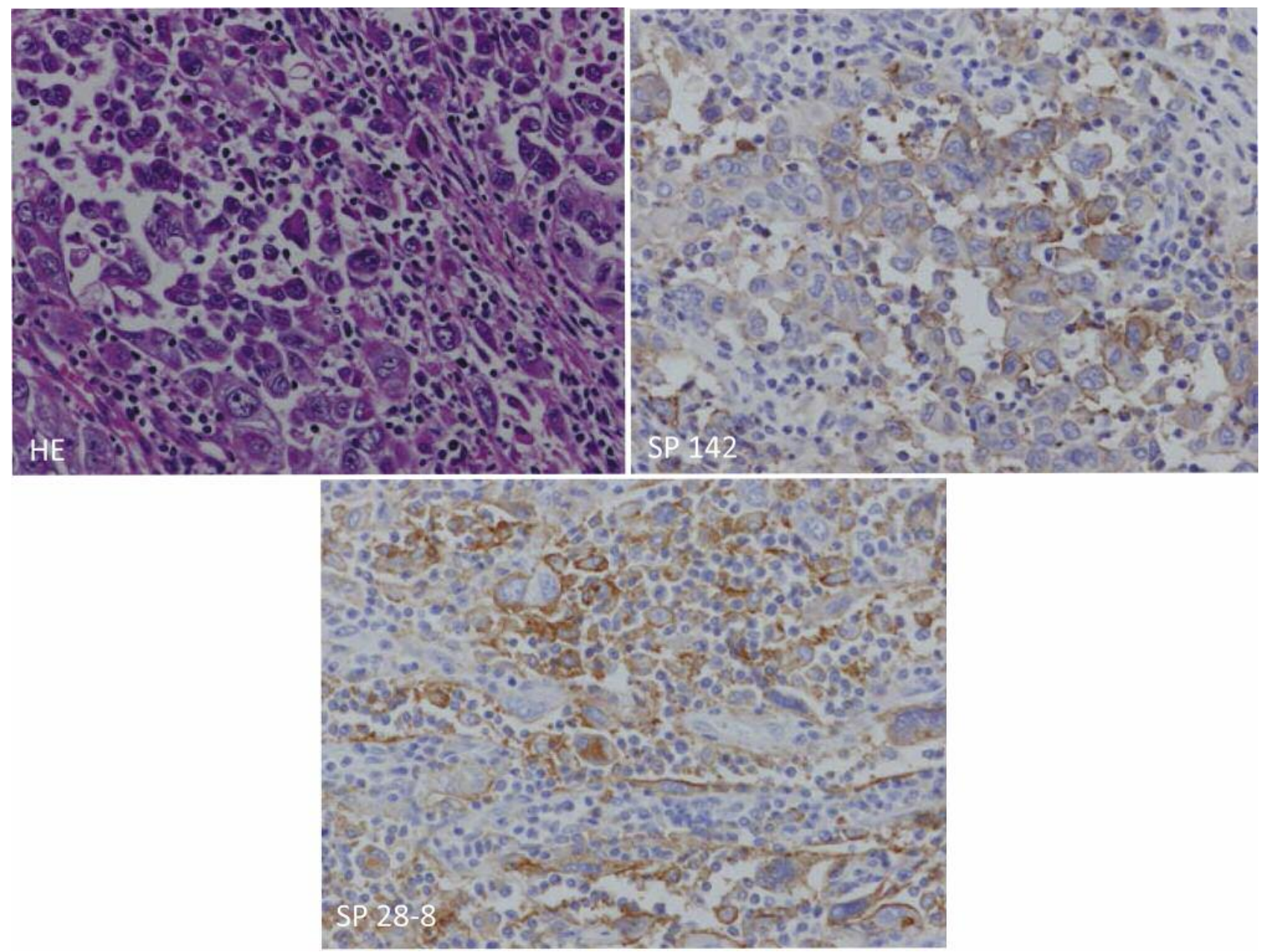

Figure 4. Haematoxylin and eosin $(H \& E)$ staining and immunohistochemistry for programmed cell death ligand 1 using clones SP142 and SP28-8 for the specimen with the highest $H$-score in this cohort.

The characteristics of the 83 enrolled patients of the validation cohort are shown in Table II. The median score and range of PD-L1 expression was 3 (0-91) and $9(0-150)$ for the SP142 and SP28-8 clones, respectively. There was no difference in $\mathrm{H}$-score between the two cohorts by $t$-test, regardless of the antibody clone used (Figure 3). Representative staining of tissue with the highest $\mathrm{H}$-score in this study (88 for SP142 and 134 for SP28-8) is shown in Figure 4.

Among the 17 patients of Cohort 1, the median duration of PFS of patients that were PD-L1-negative was significantly longer than that of those that were PD-L1positive (Figure 5A and B). A PD-L1 H-score $>5$ using the SP28-8 clone (hazard ratio(HR) $=6.46,95 \%$ confidence interval $(\mathrm{CI})=1.209-34.53 ; \quad p=0.029) \quad$ was a significant negative factor that affected the duration of PFS following curative operation or chemoradiation. Multivariate analysis could not be carried out because of the small sample size.

\section{Discussion}

In this study, we evaluated whether irradiation in patients with NSCLC induces the expression of PD-L1 on tumor cells but instead found a reduction in PD-L1 expression after induction chemoradiotherapy.

This result was unlike a model proposed in a previous report (4), in which expression of PD-L1 was up-regulated in the tumor and tumor microenvironment after ionizing irradiation. However, this finding is in line with the "abscopal effect" (8), which is the concept that localized radiation therapy is able to elicit out-of-target tumor responses. This process is likely mediated by the immune system, with dendritic cells, T-regulatory cells and suppressor cells acting as critical mediators (9-11). Our result may demonstrate that PD1/PD-L1 immunomodulation contributes to this abscopal effect (12). 
A

A

1.0

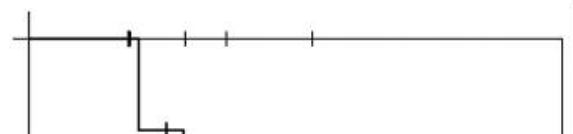

\begin{tabular}{|ccccc|}
\hline SP28-8 & $\begin{array}{c}\text { No. of } \\
\text { patients }\end{array}$ & Censored & $\begin{array}{c}\text { Median } \\
\text { PFS }\end{array}$ & $95 \% \mathrm{Cl}$ \\
\hline$<5$ & 9 & 5 & 1303 & $857-\mathrm{NA}$ \\
$>5$ & 8 & 3 & 369 & $176-\mathrm{NA}$ \\
\hline
\end{tabular}

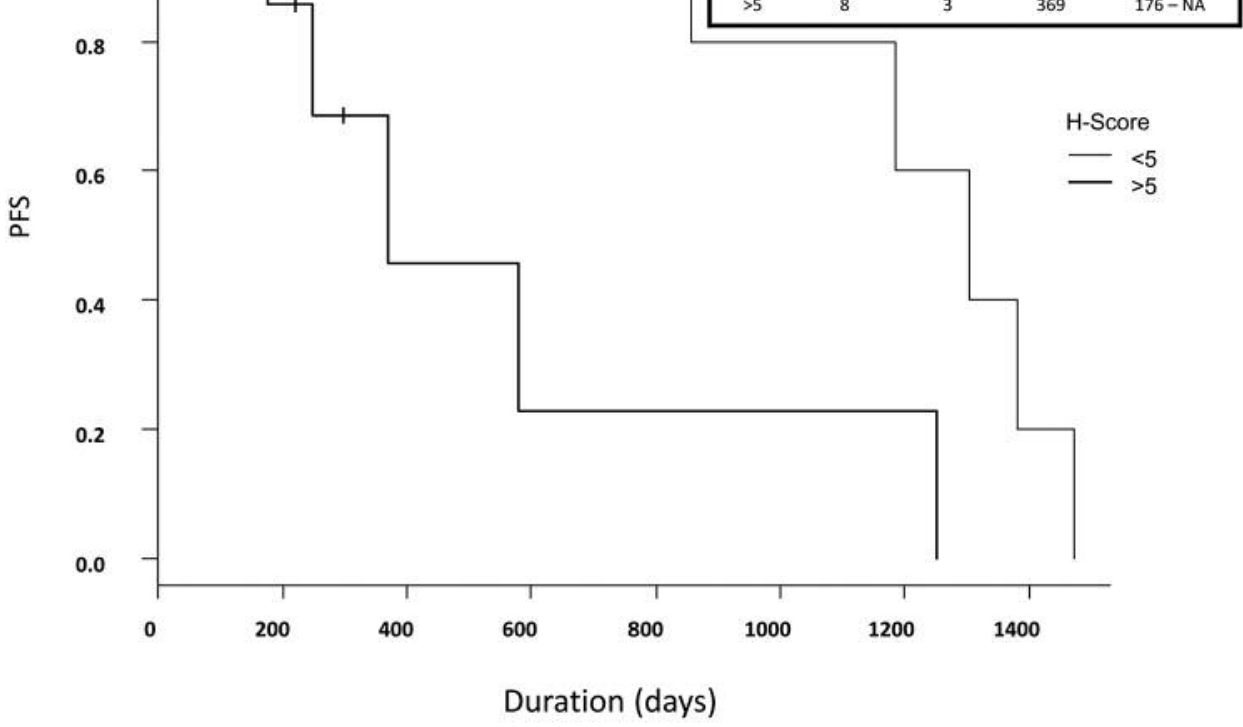

B

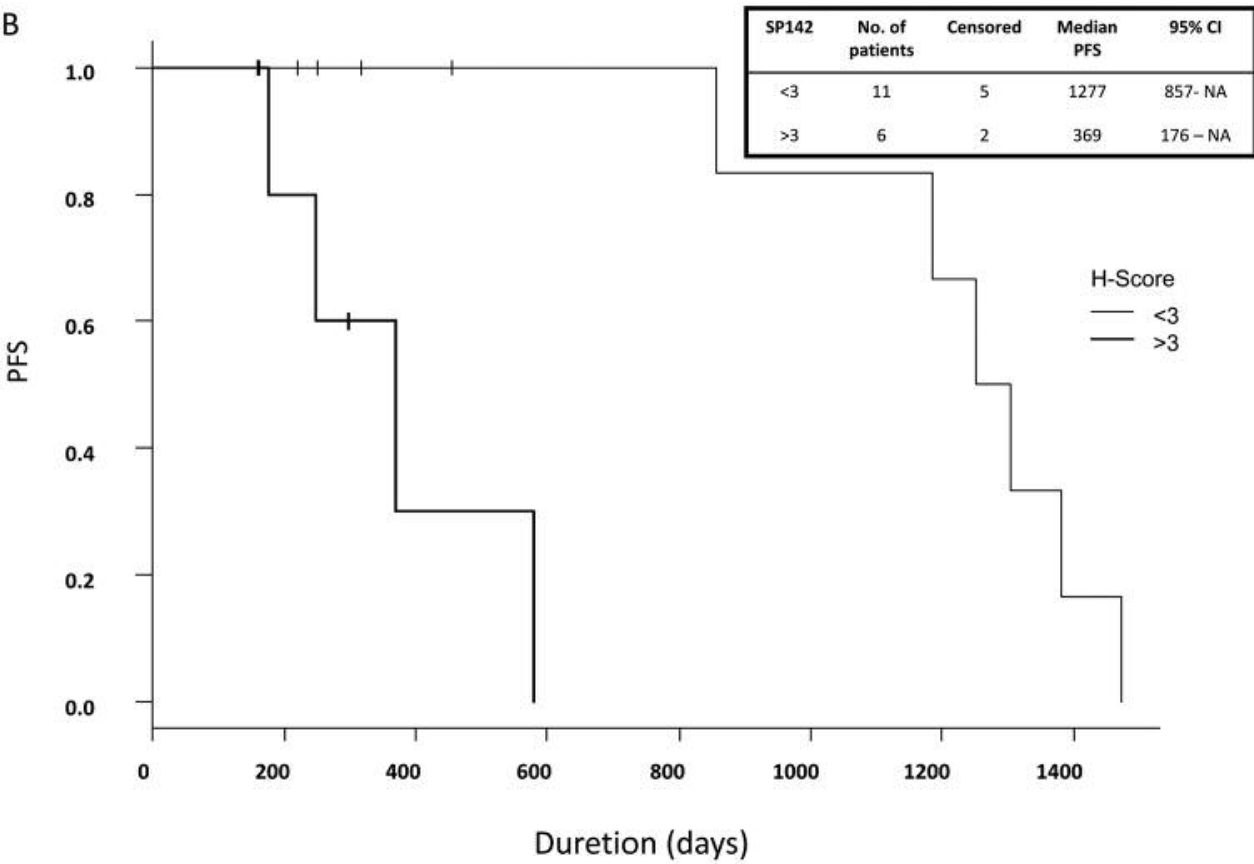

Figure 5. Progression-free survival (PFS) by Kaplan-Meier analysis of 17 patients was related to their post-therapy H-Score for programmed cell death ligand 1 (PD-L1) using clones SP28-8 (A) and SP142 (B). The difference between the groups was evaluated with the log-rank test (A: $p=0.0138 ; B: p=0.0046)$. NA: Not applicable.

The notion that local up-regulation of the PD1-PD-L1 axis suppresses radiation-induced immune responses and limits the full expression of antitumor immunity thereby facilitating relapse may be correct. However, it may depend on baseline expression of the PD1-PD-L1 axis, not on expression changes induced by ionizing irradiation. This is corroborated by our finding that PFS was significantly longer in PD-L1-negative patients. In other words, ionizing irradiation itself might be an immunomodulator that induces peripheral T-cell repertoires.

Many studies to confirm biomarkers of the response to 
drugs targeting the PD1-PD-L1 axis are currently being performed. For instance, anti-PD1 therapy has been reported to be correlated with the nonsynonymous mutation burden in NSCLC (13). However, it is currently unclear whether the first candidate biomarker, immunological analysis of PD-L1, should be an absolute selection criterion for therapy. Sunshine et al. reported that the response rate to PD-L1 blockade in patients with PD-L1-positive tumors was $48 \%$ compared to $15 \%$ in those with PD-L1-negative tumors (14). According to these results, additional information, such as genetic backgrounds and change in gene expression after ionizing irradiation, will benefit this field of study.

The limitations of our study include a small sample size and the retrospective nature of the study. Nonetheless, we were able to show a reduction in PD-L1 expression after induction chemoradiotherapy, albeit from samples of only six patients, and a genuine up-regulation of the PD1-PD-L1 axis that may be a factor in therapeutic resistance. A prospective study with stricter criteria for the selection of patients is needed to overcome these limitations.

In conclusion, we showed that tumor PD-L1 expression in patients with NSCLC who received chemoradiotherapy decreased and radiation resistance might be due to pre-treatment expression of PD-L1. This phenomenon provides a rationale for the use of immune checkpoint inhibitors as combination therapy with irradiation or adjuvant therapy for NSCLC.

\section{Compliance with Ethical Standards}

All procedures performed in studies involving human participants were in accordance with the ethical standards of the Institutional Committee and with the 1964 Helsinki declaration and its later amendments or comparable ethical standards.

\section{Funding}

None.

\section{Conflicts of Interest}

The Authors declare they have no conflicts of interest in regard to this study.

\section{Acknowledgements}

PD-L1 IHC was performed by GeneticLab Co., Ltd, Japan.

\section{References}

1 Gridelli C, de Marinis F, Cappuzzo F, Di Maio M, Hirsch FR, Mok T, Morgillo F, Rosell R, Spigel DR, Yang JC and Ciardiello F: Treatment of advanced non-small-cell lung cancer with epidermal growth factor receptor (EGFR) mutation or $A L K$ gene rearrangement: results of an international expert panel meeting of the Italian Association of Thoracic Oncology. Clin Lung Cancer 15: 173-181, 2014.
2 Jing W, Li M, Zhang Y, Teng F, Han A, Kong L and Zhu H: PD1/PD-L1 blockades in non-small-cell lung cancer therapy. Onco Targets Ther 9: 489-502, 2016.

3 Champiat S, Ileana E, Giaccone G, Besse B, Mountzios G, Eggermont A and Soria JC: Incorporating immune-checkpoint inhibitors into systemic therapy of NSCLC. J Thorac Oncol 9: 144-153, 2016.

4 Deng L, Liang H, Burnette B, Beckett M, Darga T, Weichselbaum RR and $\mathrm{Fu}$ YX: Irradiation and anti-PD-L1 treatment synergistically promote antitumor immunity in mice. J Clin Invest 124: 687-695, 2014.

5 Hata A, Fujita S, Kaji R, Katakami N and Imai Y: Do complex mutations of the epidermal growth factor receptor gene reflect intratumoral heterogeneity? J Thorac Oncol 6: 1144-1146, 2011.

6 Choi YL, Takeuchi K, Soda M, Inamura K, Togashi Y, Hatano S, Enomoto M, Hamada T, Haruta H, Watanabe H, Kurashina K, Hatanaka H, Ueno T, Takada S, Yamashita Y, Sugiyama Y, Ishikawa $\mathrm{Y}$ and Mano H: Identification of novel isoforms of the EML4-ALK transforming gene in non-small cell lung cancer. Cancer Res 68: 4971-4976, 2008.

7 Fukui T, Yatabe Y, Kobayashi Y, Tomizawa K, Ito S, Hatooka S, Matsuo K and Mitsudomi T: Clinicoradiologic characteristics of patients with lung adenocarcinoma harboring EML4-ALK fusion oncogene. Lung Cancer 77: 319-325, 2012.

8 Nobler MP: The abscopal effect in malignant lymphoma and its relationship to lymphocyte circulation. Radiology 93: 410-412, 1969.

9 Demaria S, Ng B, Devitt ML, Babb JS, Kawashima N, Liebes L and Formenti SC: Ionizing radiation inhibition of distant untreated tumors (abscopal effect) is immune mediated. Int $\mathbf{J}$ Radiat Oncol Biol Phys 58: 862-870, 2004.

10 Formenti SC and Demaria S: Systemic effects of local radiotherapy. Lancet Oncol 10: 718-726, 2009.

11 Postow MA, Callahan MK, Barker CA, Yamada Y, Yuan J, Kitano S, Mu Z, Rasalan T, Adamow M, Ritter E, Sedrak C, Jungbluth AA, Chua R, Yang AS, Roman RA, Rosner S, Benson B, Allison JP, Lesokhin AM, Gnjatic S and Wolchok JD: Immunologic correlates of the abscopal effect in a patient with melanoma. N Engl J Med 366: 925-931, 2012.

$12 \mathrm{Ng} \mathrm{J}$ and Dai T: Radiation therapy and the abscopal effect: a concept comes of age. Ann Transl Med 4: 118, 2016.

13 Rizvi NA, Hellmann MD, Snyder A, Kvistborg P, Makarov V, Havel JJ, Lee W, Yuan J, Wong P, Ho TS, Miller ML, Rekhtman N, Moreira AL, Ibrahim F, Bruggeman C, Gasmi B, Zappasodi R, Maeda Y, Sander C, Garon EB, Merghoub T, Wolchok JD, Schumacher TN and Chan TA: Cancer immunology. Mutational landscape determines sensitivity to PD-1 blockade in non-small cell lung cancer. Science 348: 124-128, 2015.

14 Sunshine J and Taube JM: PD-1/PD-L1 inhibitors. Curr Opin Pharmacol 23: 32-38, 2015. 